\title{
Improving the Peri-operative care of Patients by instituting a 'Block Room' for Regional Anaesthesia
}

\author{
Maria Chazapis, Navkiran Kaur, Damon Kamming \\ University College Hospital, London
}

\begin{abstract}
Regional anaesthesia has multiple known benefits over general anaesthesia alone, but requires time and expertise for its application. This study aimed to decrease anaesthetic time and increase total surgical operative time by instituting a 'block room' where regional anaesthesia nerve blocks could be provided by expert anaesthetists in regular scheduled sessions.

A baseline audit showed that 2 hours per day was spent on performing nerve blocks. Development of the block room allowed nerve blocks to be performed in parallel to surgical operations, reducing the mean anaesthetic control time from 44 mins to 27 mins. This freed time for an extra operative case per day. In addition, pooling of expertise to one site has allowed excellent teaching opportunities for anaesthetic trainees, and a specific training programme for regional anaesthesia is being produced.
\end{abstract}

In conclusion, instituting a block room has improved the efficiency of our theatre complex, and improved the service deliverable to our patients.

\section{Problem}

Providing nerve blocks (regional anaesthesia) for surgical patients for anaesthesia and post-operative analgesia has significant outcome benefits. These include increased patient satisfaction [1], improved quality of recovery, decreased opioid use and decreased length of stay in recovery. In addition, latest research shows possible decreased cancer recurrence after breast surgery [2][3].

Ultrasound-guided nerve blocks are undertaken by experienced and skilled operators. However, use of regional anaesthesia can add significant time to the provision of anaesthesia, reducing the available operative time for surgery. If an anaesthetist is not confident in providing nerve blocks, or is constrained by time limitations, then patients are not being offered the best analgesia and peri-operative experience available.

\section{Background}

Regional anaesthesia is known to decrease recovery times, decrease post-operative nausea, decrease post-operative pain, and increase patient satisfaction [4]. However, expertise and time is needed to perform nerve blocks. A patient's pathway through theatres and surgery is very linear, starting off in the admissions lounge, moving to a holding bay, the anaesthetic room for their anaesthesia, the operating room for their surgery and then moving to the recovery area for recovery and discharge home or to a ward. At any of these locations unnecessary delays lead to decreased theatre efficiency.

Block room models have been in use for the past decade in North America, but are only a recent introduction in the UK. Allowing nerve blocks to be performed in a separate site whilst other patients are being operated on in theatre, reduces anaesthetic room time and can potentially increase time available for surgical procedures. This changes the patient's pathway through theatres from a linear model to a 'parallel performance' model. Indeed, creating a block room for hand surgery enabled surgeons in Vancouver to operate on an extra 3 patients per day [5]. Data from Sunnybrook in Toronto suggests an $18 \%$ reduction in turnover time, leading to an extra joint arthroplasty per day [6].

Regional anaesthesia is known to decrease time patients spend in recovery. This is attributed to decreased pain scores and decreased nausea and vomiting, reducing recovery staff workload [7]. There is potential to fast-track patients directly to second stage recovery, reducing the bottleneck of first stage recovery, which is currently a common inefficiency.

Pooling resources into the block room would lead to a reduced failure rate of regional anaesthesia by standardising ultrasound guidance (NICE guidance), and allowing time to test and top up ineffective blocks. Effective regional anaesthesia prior to surgery results in a cost saving of anaesthetic and analgesic drugs costs of up to $50 \%$ [8].

Teaching and training with the block room model would greatly improve the safety of a regional anaesthesia service. Focused periods of time in a block room enables more consistent training as experienced by trainees returning from regional fellowships in North America where the model is standard. We would be one of the first hospitals in the United Kingdom to develop such an innovative service. Ultimately the patient would benefit from the improved focused clinical care from a dedicated team. Guidelines and protocols for specific procedures would be established. The WHO Safer Surgery Checklist would focus our improved teamwork directly onto the patient. 


\section{Baseline measurement}

An initial audit was undertaken to measure how many nerve blocks were being performed in theatres, and to measure how long each one took. The results showed that on average, 25 nerve blocks were being performed each week, averaging 5 blocks a day. Each day, 120 minutes were spent on these nerve blocks, averaging 18 minutes each. The average time in the anaesthetic room before the patient was ready for surgery was 44 minutes. These results show that significant time is used every day for the provision of regional anaesthesia. More efficient use of this time could lead to an increase in time available to perform surgical procedures with the potential to avoid surgical cancellations and to allow booking of extra surgical cases.

\section{Design}

Our intervention was to develop a 'block room' to improve theatre efficiency through parallel processing. We converted an unused part of our recovery space into a dedicated block room, and staffed it daily with one expert consultant anaesthetist, a regional anaesthesia fellow, an anaesthetic trainee and a senior operating department practitioner.

On the morning of surgery, potential patients are identified for nerve blocks. After agreement with the operating team, consent from the patient is obtained, and the patient is brought to the block room early for their nerve block. The patient then remains in the block room until their operating theatre is ready. This has enabled parallel performance of regional anaesthesia whilst surgery is ongoing. In addition, pooling of expertise (trained staff) and equipment (ultrasound machines), results in increased efficiency, and provide valuable teaching and training opportunities for trainees.

\section{Strategy}

Timings of the patient's pathway through the theatre suite, and types of nerve block performed were continually recorded. Plan-dostudy-act (PDSA) cycles were completed at each weekly assessment.

In week 1, there was some confusion on the patient's pathway, which was addressed by teaching sessions and communication with all staff involved. In week 2 , there were some theatre delays in the morning because patients who were first on the theatre list were not being brought up to the block room early enough. The problem was discussed with the surgical reception staff, and we devised a system of flagging patients eligible for the block room, ensuring those patients are prepared for theatre first. Since this change, the patient pathway has become well established, and staff have maintained patient flow.

One continuing problem is staff shortages secondary to sickness. Approximately once every fortnight, our operating department practitioner (ODP) is re-allocated to a theatre list. We have decided it is unsafe to run a block room without an experienced ODP, as we often have two sedated, blocked patients waiting for their surgery, and local anaesthetic toxicity is an exceedingly rare but possibly life threatening complication. However, on these days, we transfer to a 'roaming block room' model, where we perform blocks in theatre anaesthetic rooms, using the theatre ODPs, whilst operations are being carried out. We cannot perform as efficiently, but we still provide the best service we can.

\section{Results}

To date, 363 peripheral nerve blocks have been performed in the block room. 5 more blocks are being performed each week (30 vs 25). The block room has reduced the mean anaesthetic room control time from 44 minutes pre block room to 27 minutes post block room. Many types of nerve blocks were performed, ranging from common interscalene, supraclavicular, ankle and transversus abdominus plane blocks, to more challenging blocks such as sciatic popliteals and paravertebrals.

See Picture 1 in the supplementary file.

The main change has been the increased number of ultrasoundguided paravertebral blocks. These are recognised as the most challenging ultrasound-guided blocks, the target endpoint being located very deep and very close to the pleura. Interest in this block has increased in breast surgery, as early research results show that it may reduce breast cancer recurrence. Before the block room, lack of available specialists would mean we would be unable to provide this block to our breast cancer patients. Due to the parallel processing of block room use, we have managed to perform more surgical cases in 2 of the 3 operating theatres. This averages to 1 extra surgical case per day. In addition, the number of late theatre starts in each of the operating theatres has decreased from a mean of 12 to 6 per month.

We have also measured that patients with nerve blocks have a reduced length of stay in recovery, and decreased peri-operative opioid use. Additionally, we have measured patient satisfaction. Over a period of four weeks, 32 patients were contacted $24 \mathrm{~h}$ after their surgery, and asked to rate how satisfied they were with their nerve block [9]. An 11-point numerical rating scale was used $(0=$ very unsatisfied, $10=$ very satisfied). $78.1 \%(n=25)$ gave a score of 10. $96.9 \%(n=31)$ gave a score equal or greater than 7 . One patient was unsatisfied because they had a failed nerve block; we always mention this is a possibility when taking consent. $92.8 \%$ of the patients would have a block again, and they would recommend it to their family and friends.

See supplementary file: ds3095.png - "Nerve Block types performed"

\section{Lessons and limitations}

In the first weeks of implementing the project, great care was undertaken to communicate clearly with the staff in theatres, anaesthetists, surgeons and the patients themselves regarding this new venture. One of the main difficulties is ensuring that patients were brought up to the block room, and not the holding-bay or 
anaesthetic rooms. Skilled operating department practitioner (ODP) assistance is essential for the efficient running of a block room list, as nerve blocks are technically challenging. There also exists a discontinuity of care between the anaesthetist delivering the nerve block and the anaesthetist delivering the intra-operative anaesthetic. As such we are currently developing a patient information leaflet for the block room experience, and a separate consent form specific for nerve blocks.

\section{Conclusion}

In summary, establishment of the block room has increased the number and type of nerve blocks performed, with a reduction in anaesthetic time in the theatres serviced, leading to reduced late start times, and an increase in the number of surgical cases performed. In addition, patients with nerve blocks have a reduced length of stay in recovery, and decreased peri-operative opioid use.

\section{References}

1. Webster F, Bremner S, McCartney CJ. Patient experiences as knowledge for the evidence base: A qualitative approach to understanding patient experiences regarding the use of regional anaesthesia for hip and knee arthroplasty. Reg Anesth Pain Med 2011;36(5):461-5

2. Exadaktylos AK, Buggy DJ, Moriarty DC, Mascha E, Sessler DI. Can anesthetic technique for primary breast cancer surgery affect recurrence or metastasis Anesthesiology 2006, Oct;105(4):660-664

3. Regional Anaesthesia and Breast Cancer Occurrence Clinical Trials Register. National Cancer Institute.

4. Liu SS, Strodtbeck WM, Richman JM, Wu CL. A Comparison of Regional Versus General Anesthesia for Ambulatory Anesthesia: A Meta-Analysis of Randomized Controlled Trials. Anesth Analg. 2005 Dec;101(6):1634-42.

5. Head SJ, Seib R, Osborn JA, Schwarz SK. A 'swing room' model based on regional anesthesia reduces turnover time and increases case throughput. Can J Anaesth 2011, Aug:58(8):725-32

6. Mariano ER, Chu LF, Peinado CR, Mazzei WJ. Anesthesiacontrolled time and turnover time for ambulatory upper extremity surgery performed with regional versus general anaesthesia. J Clin Anesth 2009, Jun;Jun21(4):253-7

7. Borgeat A, Ekatodramis G, Schenker CA. Postoperative nausea and vomiting in regional anesthesia: $A$ review. Anesthesiology 2003,Feb;98(2):530-47

8. Gonano C, Kettner SC, Ernstbrunner M, Schebesta K, Chiari A, Marhofer P. Comparison of economical aspects of interscalene brachial plexus blockade and general anaesthesia for arthroscopic surgery. Br J Anaesth 2009,Sep;103(3):428-3

9. Sebastian MP, Kaur N, Kamming D. Can regional anaesthesia be performed before general anaesthesia without compromising patient satisfaction: patient's perspective in a central London teaching hospital. Poster, ASRA Chicago 2013.

\section{Declaration of interests}

Nothing to declare

\section{Acknowledgements}

Dr Simeon West, Dr Sanjiv Patel 